\title{
New approaches to EGFR inhibition for locally advanced or metastatic squamous cell carcinoma of the head and neck (SCCHN)
}

\author{
Mark Agulnik
}

Received: 23 December 2011/ Accepted: 4 January 2012/Published online: 18 January 2012

(C) The Author(s) 2012. This article is published with open access at Springerlink.com

\begin{abstract}
Despite recent advances in radiotherapy and chemotherapy, survival rates for squamous cell carcinoma of the head and neck (SCCHN) have remained poor. The focus of SCCHN therapy has more recently shifted to the molecular level, particularly the epidermal growth factor receptor (EGFR/ErbB) pathway. Several agents that target the EGFR pathway, including monoclonal antibodies and tyrosine kinase inhibitors, are under investigation for SCCHN. Searches of PubMed and results of key oncology congresses were performed to identify relevant articles and abstracts. The EGFR-targeted monoclonal antibody cetuximab is approved for the treatment of locally advanced SCCHN in combination with radiotherapy, for first-line treatment of recurrent or metastatic SCCHN in combination with platinum-based chemotherapy and 5-fluorouracil, and for recurrent or metastatic SCCHN following progression with platinum-based chemotherapy. Other investigational EGFR-targeted monoclonal antibodies (e.g., panitumumab, nimotuzumab, zalutumumab) are in clinical development for SCCHN. Inhibition of the tyrosine kinase domain of EGFR has also been explored as a therapeutic approach in SCCHN using small-molecule reversible inhibitors, such as gefitinib and erlotinib. However, a key challenge in SCCHN is the development of resistance, and strategies are being pursued to delay or overcome resistance to EGFR-targeted agents. These strategies include development of agents that inhibit multiple ErbB receptors simultaneously (e.g., lapatinib) or that bind multiple ErbB
\end{abstract}

\footnotetext{
M. Agulnik ( $\bowtie)$

Department of Medicine, Division of Hematology/Oncology, Northwestern University Feinberg School of Medicine, 676 North Saint Clair Street, Suite 850, Chicago, IL 60611-2942, USA

e-mail: m-agulnik@northwestern.edu
}

family receptors irreversibly (e.g., afatinib, PF-00299804) and investigation of combinations of agents that target multiple pathways implicated in the pathogenesis of SCCHN. Ongoing large clinical trials are evaluating these emerging agents and combinations for the treatment of SCCHN.

Keywords Epidermal growth factor receptor (EGFR) . Monoclonal antibody - Tyrosine kinase inhibitor .

Resistance $\cdot$ Squamous cell carcinoma of the head and neck

\section{Introduction}

Head and neck cancer comprises a spectrum of malignancies that develop primarily within the oral cavity, pharynx, and larynx. In 2010, there were an estimated 49,260 new cases of head and neck cancers in the United States, and 11,480 patients died from the disease [1]. Squamous cell carcinoma of the head and neck (SCCHN) is the principal histologic subtype of this disease, accounting for $>90 \%$ of all cases [2]. Management of early-stage SCCHN often involves surgery or radiotherapy, which may be curative. Locally advanced disease is generally treated with a combination of surgery, chemoradiotherapy, and/or targeted therapy, while the standard approach for treatment of recurrent or metastatic SCCHN is platinum-based chemotherapy [3, 4].

The focus of SCCHN therapy has shifted to the molecular level, particularly the epidermal growth factor receptor (EGFR/ErbB1/HER1) pathway (Fig. 1) [5]. EGFR protein expression is detected in $>90 \%$ of all SCCHN tumors [6-8]. In addition, high levels of EGFR protein expression and increased EGFR gene copy number are associated with decreased survival [7-12], resistance to 


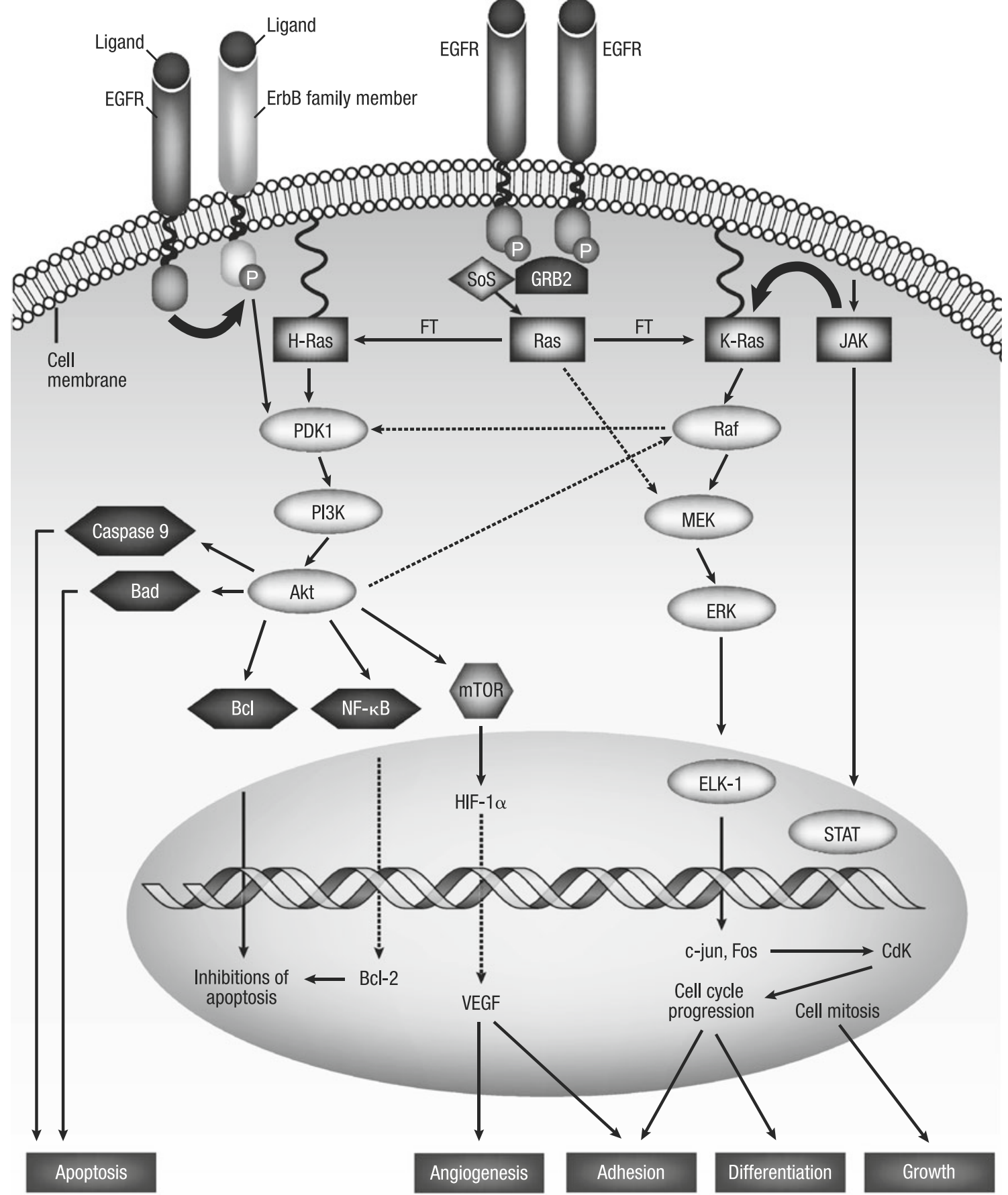

Fig. 1 Epidermal growth factor receptor and ErbB family downstream signaling pathways potentially involved in squamous cell carcinomas of the head and neck. Downstream pathways activated by dimerization and activation of the ErbB family. Adapted with permission from Venook et al. [5]. (C2005 John Wiley \& Sons, Inc. $A k t$ v-akt murine thymoma viral oncogene homolog, serine-threonine kinase 1, Bad Bcl-2 antagonist of cell death, $B c l$ B-cell lymphoma, $C d K$ cyclin dependent kinase, $E G F R$ epidermal growth factor receptor, $E L K-1$ Ets like gene 1, ErbB erythroblastic leukemia viral oncogene homolog, ERK extracellular signal-regulated kinase, Fos protooncogene c-fos, GRB2 growth factor receptor-bound protein 2, $H I F-1 \alpha$ hypoxia inducible factor- $1 \alpha$, JAK Janus kinase, MEK mitogen-activated protein kinase kinase, mTOR mammalian target of rapamycin, $N F-\kappa B$ nuclear factor- $\kappa \mathrm{B}, P I 3 K$ phosphatidylinositol-3kinase, Raf v-raf 1 murine leukemia viral oncogene homolog 1, Ras retrovirus-associated DNA sequences, $S o S$ son of sevenless, STAT signal transducers and activators of transcription, $V E G F$ vascular endothelial growth factor 
radiotherapy [13], locoregional treatment failure [7-9], and increased rates of distant metastases [8, 14].

Cetuximab (Erbitux ${ }^{\circledR}$, Bristol-Myers Squibb; New York, NY, USA), a recombinant chimeric anti-EGFR monoclonal antibody (mAb), was the first molecularly targeted therapy approved for SCCHN. Cetuximab is approved in combination with radiation therapy for locally advanced disease, in combination with platinum-based chemotherapy and 5-fluorouracil (5-FU) for the first-line treatment of metastatic/recurrent disease, and as a single agent for metastatic/recurrent disease after failure of platinum-based chemotherapy [15]. This article will briefly review the clinical trial data associated with cetuximab in SCCHN, describe limitations of current therapy, and discuss data associated with investigational EGFR- and ErbB family targeted treatment strategies for SCCHN.

\section{Cetuximab: proof of concept of EGFR inhibition in locally advanced or metastatic SCCHN}

Results from several clinical trials have established the activity of cetuximab in the treatment of SCCHN. A landmark phase III study involving 424 patients with locoregionally advanced SCCHN compared cetuximab in combination with high-dose radiotherapy versus high-dose radiotherapy alone [16]. The combination of cetuximab and radiotherapy significantly improved median overall survival (OS; 49.0 vs. 29.3 months; hazard ratio [HR], $0.74 ; 95 \%$ confidence interval $[\mathrm{CI}], 0.57-0.97 ; P=0.03$ ) and median progression-free survival (PFS; 17.1 vs. 12.4 months; HR, 0.70; 95\% CI, 0.54-0.90; $P=0.006$ ) versus radiotherapy alone. The three most common adverse events (AEs; $\geq 10 \%$ of patients) were mucositis (93\% for cetuximab/radiotherapy vs. $94 \%$ for radiotherapy alone), radiation dermatitis ( $86 \%$ vs. $90 \%$ ), and acneiform rash ( $87 \%$ vs. $10 \%$ ). The incidence of grade $\geq 3$ AEs was also similar between groups, with the exception of acneiform rash $(17 \%$ with cetuximab/radiation vs. $1 \%$ with radiation alone; $P<0.001)$ and infusion-related events (3\% vs. $0 \%$; $P=0.01$ ) [16]. Notably, incidence of radiation-associated acute toxicities was not increased in combination with cetuximab. Five-year survival rates were $45.6 \%$ for cetuximab/radiation versus $36.4 \%$ for radiation alone (HR, $0.73 ; 95 \%$ CI, 0.56-0.95; $P=0.018)$ [17]. Preliminary results from the phase III RTOG 0522 trial $(N=940)$ evaluating concurrent accelerated radiation/cisplatin with or without cetuximab demonstrated no significant improvement with cetuximab in PFS (HR, 1.05; 95\% CI, $0.84-1.29 ; P=0.66$ ), OS (HR, 0.87; 95\% CI, 0.66-1.15; $P=0.17)$, or total grade $\geq 3$ AEs $(92 \%$ vs. $90 \%$; $P=0.30)$. Higher rates of grade 3-4 mucositis (45\% vs. $35 \% ; P=0.003)$ and skin reactions $(40 \%$ vs. $17 \%$;
$P<0.0001)$ were observed in the cetuximab arm [18]. Cetuximab has also been evaluated in phase II studies in combination with cisplatin and radiation in patients with locally advanced SCCHN $[19,20]$.

In a number of ongoing phase III studies, cetuximab is being evaluated in patients with locally advanced SCCHN. Induction chemotherapy with docetaxel/cisplatin/5-FU (TPF) followed by cisplatin/radiotherapy versus cetuximab/ radiotherapy (NCT00716391); cetuximab/radiotherapy versus cisplatin/radiotherapy (NCT00999700); and cetuximab/radiotherapy versus carboplatin/5-FU/radiotherapy (NCT01233843) is also being investigated. Another phase III trial is evaluating TPF followed by radiotherapy plus either concomitant chemotherapy or concomitant cetuximab versus radiotherapy plus either concomitant chemotherapy or concomitant cetuximab, without induction TPF (NCT01086826).

Several clinical trials have evaluated cetuximab in the metastatic/recurrent SCCHN setting. In the phase III E5397 trial $(N=117)$, first-line treatment with cisplatin plus cetuximab significantly improved response rate (RR) versus cisplatin alone ( $26 \%$ vs. $10 \% ; P=0.03$ ) [21]. However, this regimen did not demonstrate a significant effect on median PFS (4.2 vs. 2.7 months; $P=0.09$ ) or median OS (9.2 vs. 8 months; $P=0.21)$. The three most common grade $3-4$ AEs ( $\geq 10 \%$ of patients) were fatigue ( $17 \%$ for cetuximab plus cisplatin vs. $14 \%$ for cisplatin alone), nausea (24\% vs. $19 \%$ ), and vomiting (17\% in each arm). In another phase III trial (EXTREME; $N=442$ ), patients with previously untreated metastatic/recurrent SCCHN were assigned randomly to receive cisplatin or carboplatin plus 5-FU and cetuximab or chemotherapy (platinum plus 5-FU) alone [22]. The addition of cetuximab to chemotherapy significantly increased median OS (10.1 vs. 7.4 months; HR, 0.80; 95\% CI, 0.64-0.99; $P=0.04$ ), median PFS (5.6 vs. 3.3 months; HR, 0.54 ; 95\% CI, 0.43-0.67; $P<0.001$ ), and RR (36\% vs. $20 \%$; odds ratio [OR], $2.33 ; 95 \%$ CI, $1.50-3.60 ; P<0.001)$ versus chemotherapy alone. The three most frequently reported grade $3-4$ AEs $(\geq 10 \%$ of patients) were neutropenia (22\% for chemotherapy/cetuximab vs. $23 \%$ for chemotherapy alone), anemia (13\% vs. $19 \%$ ), and thrombocytopenia (11\% vs. $11 \%$ ) [22]. A separate phase II study evaluated cetuximab in combination with carboplatin/paclitaxel as first-line therapy in patients who had previously received induction or adjuvant platinumbased chemotherapy $(N=33)$ [23]. The RR was $46 \%$, median OS was 10.3 months.

Two phase II studies demonstrated the efficacy of cetuximab in combination with platinum-based chemotherapy as second-line treatment in patients with metastatic/recurrent SCCHN who failed to respond to first-line platinum-based chemotherapy alone [24, 25]. Reported RRs and median OS were approximately $10 \%$ and 
5-6 months, respectively, and the most common AEs were anemia, rash, asthenia, and nausea/vomiting. In another phase II trial in patients with metastatic/recurrent SCCHN refractory to platinum-based chemotherapy $(N=103)$, cetuximab monotherapy was associated with a RR of $13 \%$ and median OS of 178 days [26]. Similar to other trials, the most common AEs were rash, acne, and asthenia.

In general, AEs associated with cetuximab are mild to moderate and clinically manageable [27]; the most common toxicity associated with cetuximab therapy is an acnelike pustular rash, which is observed in $>70 \%$ of patients $[16,21,24]$. In some studies, an association between the presence of rash and improved OS has been proposed [17, 21, 24]. Hypomagnesemia may also occur following cetuximab therapy (reported in 5-14\% of patients) [21, 22], due to inhibition of magnesium reabsorption within the kidney secondary to EGFR blockade [27, 28]. Patients therefore require routine monitoring during treatment. Grade 4 infusion-related reactions have also been reported in a minority $(\sim 3 \%)$ of patients $[17,21,27,28]$.

\section{Limitations of current treatment options for locally advanced or metastatic SCCHN}

The current standard of care for locally advanced SCCHN may consist of surgery, chemoradiotherapy, and/or cetuximab therapy [3]. Although developments in radiotherapy and surgical and imaging techniques have improved patient function following intervention (e.g., preservation of associated and adjacent organs and structures) [29-31], OS has increased only modestly. In addition, current treatments may be associated with both acute and chronic adverse effects [29, 30]. A meta-analysis of clinical trial data from $>17,000$ SCCHN patients treated with curative intent showed that concomitant chemotherapy and radiotherapy was associated with an absolute 5-year survival benefit of $6.5 \%$ (over radiotherapy alone) compared with $2.4 \%$ for induction chemotherapy followed by radiotherapy versus radiotherapy alone [30]. In the previously mentioned landmark phase III trial in patients with locally advanced SCCHN that compared cetuximab in combination with high-dose radiotherapy versus high-dose radiotherapy alone, the 5-year survival benefit with the addition of cetuximab to radiotherapy was approximately $9 \%$ versus radiotherapy alone [17]. While this compares favorably to the $6.5 \%$ increase observed with the addition of platinumbased chemotherapy to radiotherapy [30], these results should be interpreted with caution as the study did not compare the cetuximab combination with platinum-based chemoradiotherapy [28].

For metastatic/recurrent SCCHN, the current standard of care is chemotherapy, particularly platinum-based agents with or without addition of 5-FU, with the goals of palliation of symptoms and prolongation of OS. In addition, cetuximab is currently approved as a treatment option for recurrent or metastatic SCCHN as first-line therapy in combination with platinum-based chemotherapy or for patients progressing after platinum-based therapy [15]. The use of combination chemotherapy in metastatic/recurrent SCCHN is based on improvement in response observed with combination chemotherapy versus single-agent chemotherapy in randomized trials [32, 33]; however, no significant extension in OS was observed. No specific doublet regimen has demonstrated improved efficacy over others to date [32, 34] (Fig. 2a), and a variety may be utilized in clinical practice [29]. The addition of a third cytotoxic agent in this patient population may improve
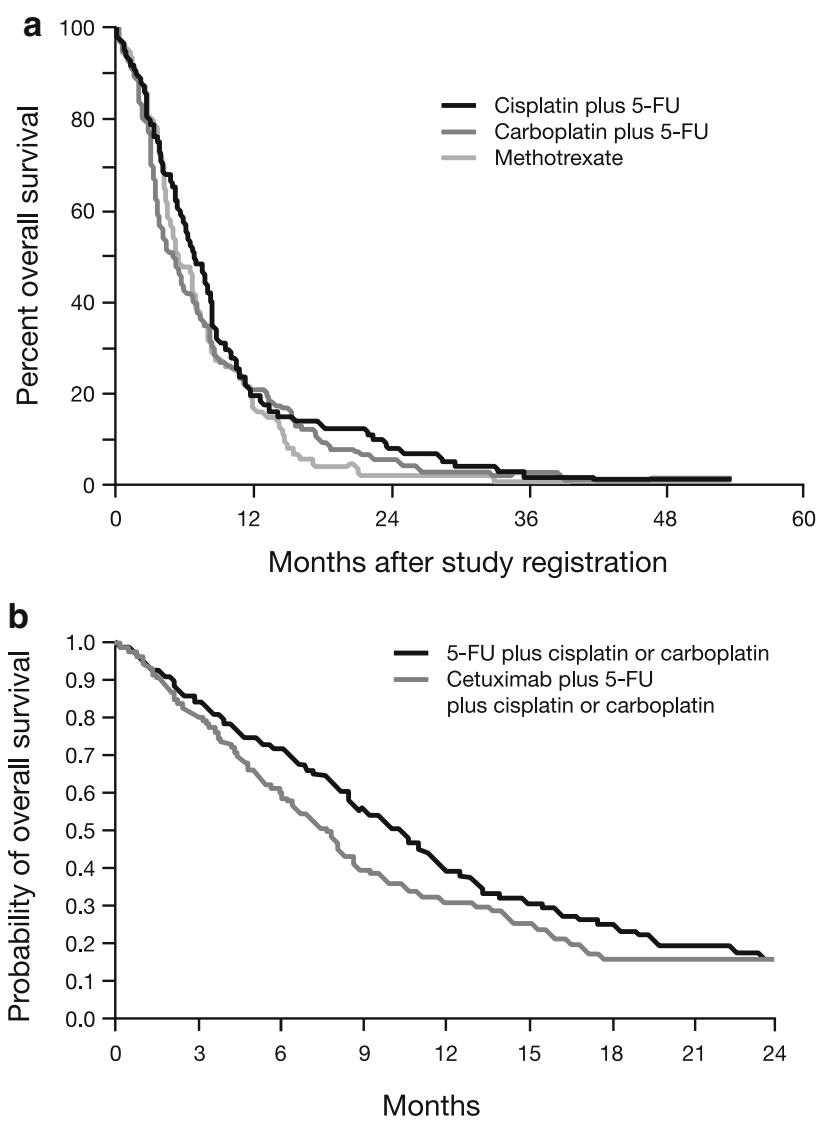

Fig. 2 Survival estimates from key trials of chemotherapy in patients with SCCHN. Kaplan-Meier estimates of overall survival in patients with metastatic/recurrent SCCHN treated with (a) methotrexate versus cisplatin/5-fluorouracil versus carboplatin/5-fluorouracil (Southwest Oncology Group) [32]; b cetuximab plus 5-fluorouracil/ platinum-based chemotherapy versus 5-fluorouracil/platinum-based chemotherapy alone (NCT00122460) [22]. a Reprinted with permission [32] (C) 1992 American Society of Clinical Oncology. All rights reserved. b Reprinted with permission [22] (C2008 Massachusetts Medical Society. SCCHN squamous cell carcinoma of the head and neck, 5-FU 5-fluorouracil 
outcomes in some cases, but this option is generally limited by increased toxicity [35-37]. The EXTREME trial was the first study in over 25 years to demonstrate a survival advantage in the metastatic/recurrent SCCHN setting, with significant improvements for cetuximab plus 5-FU and platinum-based chemotherapy versus chemotherapy alone (Fig. 2b) [22]. However, the issues of treatment sequence, potential cross-resistance, synergy, and whether the added benefit of cetuximab would be maintained if given after chemotherapy upon disease progression were not addressed [28].

Despite therapeutic advances, the 5-year survival rate for head and neck cancers in the US has remained approximately 55-65\% since the mid-1970s [28, 38]. Both radiotherapy and chemotherapeutic approaches may have been optimized in terms of balancing efficacy and safety/ tolerability [4], and the use of higher doses of chemotherapy in an attempt to overcome resistance has generally resulted in unacceptable toxicity and damage to healthy adjacent tissues [28]. While cetuximab has demonstrated activity in SCCHN, new agents and treatment strategies are needed that will provide both improved tolerability and efficacy.

\section{Future directions beyond cetuximab: inhibiting the ErbB family}

Several novel agents targeting the ErbB/HER receptor family are being evaluated in phase II and III clinical trials for the treatment of SCCHN (Table 1).

\section{Anti-EGFR monoclonal antibodies}

Panitumumab (Vectibix ${ }^{\circledR}$, Amgen; Thousand Oaks, CA, USA) is a fully human anti-EGFR mAb. In a phase I study, the combination of panitumumab with carboplatin, paclitaxel, and intensity-modulated radiotherapy was evaluated in patients with locally advanced SCCHN $(N=19)$ [39]. All patients achieved at least a partial response (PR), and the most common AEs ( $\geq 10 \%$ of patients) were oral pain, xerostomia, acneiform rash, and anemia. The phase II PRISM study evaluated second-line panitumumab monotherapy following prior chemotherapy for metastatic/ recurrent $\operatorname{SCCHN}(N=52)$ [40]. The interim safety analysis demonstrated that the most common AEs $(\geq 10 \%$ of patients) were skin disorders, fatigue, hypomagnesemia, and nausea. Grade $\geq 3$ skin-related AEs were observed in $12 \%$ of patients. In SPECTRUM, cisplatin/5-FU plus panitumumab was compared with cisplatin/5-FU alone in patients with metastatic/recurrent SCCHN $(N=657)$ [41]. The addition of panitumumab to chemotherapy did not significantly improve median OS versus chemotherapy alone (11.1 vs. 9.0 months; HR, 0.87; 95\% CI, 0.73-1.05; $P=0.14)$, but did improve median PFS (5.8 vs. 4.6 months; HR, 0.78; 95\% CI, 0.66-0.92; $P=0.004)$. The RR was $36 \%$ for panitumumab plus chemotherapy versus $25 \%$ for chemotherapy alone. The three most common grade $\geq 3$ AEs ( $\geq 10 \%$ of patients) were neutropenia (32\% for panitumumab and chemotherapy vs. 33\% for chemotherapy alone), skin toxicity (17\% vs. $1 \%$ ), and anemia (12\% vs. 14\%). Infusion-related reactions of any grade occurred in $<1 \%$ of patients in each group [41].

Several ongoing phase II studies are currently evaluating panitumumab in locally advanced SCCHN (combination with radiotherapy vs. chemoradiotherapy, NCT00547157; combination with chemoradiotherapy, NCT00500760; combination with postoperative chemoradiotherapy, NCT00798655) or metastatic/recurrent SCCHN (as first-line treatment in combination with cisplatin and docetaxel, NCT00454779). An ongoing phase III trial is evaluating panitumumab plus radiotherapy versus cisplatin plus radiotherapy for locally advanced SCCHN (NCT00820248).

Nimotuzumab (YM Biosciences; Ontario, Canada) is a humanized anti-EGFR $\mathrm{mAb}$ that has been granted approval in SCCHN in several countries outside the United States. It exhibits a reduced binding affinity for the EGFR compared with the murine mAb [42], but has demonstrated a unique clinical profile, with an absence of the severe skin toxicities that are observed with cetuximab and panitumumab.
Table 1 ErbB family inhibitors in phase II and III studies for the treatment of squamous cell carcinoma of the head and neck

$E G F R$ epidermal growth factor receptor, $I V$ intravenous, $m A b$ monoclonal antibody, $P O$ oral, TKI tyrosine kinase inhibitor

\begin{tabular}{lll}
\hline Agent & Mechanism/target/binding & $\begin{array}{l}\text { Method of } \\
\text { administration }\end{array}$ \\
\hline Panitumumab & Fully human anti-EGFR mAb & IV \\
Nimotuzumab & Humanized anti-EGFR mAb & IV \\
Zalutumumab & Fully human anti-EGFR mAb & IV \\
Gefitinib & Reversible, small-molecule EGFR TKI & PO \\
Erlotinib & Reversible, small-molecule EGFR TKI & PO \\
Lapatinib & Reversible, small-molecule EGFR/ErbB2 TKI & PO \\
Afatinib (BIBW 2992) & Irreversible, small-molecule ErbB family inhibitor & PO \\
PF-00299804 & Irreversible, small-molecule pan-HER TKI & PO \\
\hline
\end{tabular}


A pharmacodynamic study assessing the combination of nimotuzumab and radiotherapy in patients with unresectable locoregional SCCHN showed that nimotuzumab was well tolerated, with no evidence of skin rash. Nine of 10 patients achieved an objective response based on RECIST criteria [43].

In a phase I/II trial, nimotuzumab plus radiotherapy was evaluated in 24 patients with locally advanced SCCHN [44]. The RR was 50\% with doses of 50-100 mg (low dose) nimotuzumab, and $81 \%$ with $200-400 \mathrm{mg}$ (high dose) nimotuzumab. Median OS for low-dose nimotuzumab was 8.6 months, compared with 44.3 months for high-dose nimotuzumab ( $P=0.03$ for high- vs. low dose). Three-year OS rates were 16.7 and $66.7 \%$ for the low- and high doses, respectively. The most common AEs with highdose nimotuzumab were fever, hypotension, and tremors. No cases of skin rash were observed [44]. A separate phase IIb study investigated nimotuzumab plus chemoradiotherapy versus chemoradiotherapy alone (group 1), or nimotuzumab plus radiotherapy versus radiotherapy alone (group 2), as first-line therapy in 92 patients with advanced unresectable SCCHN [45]. The RR (100\% vs. $70 \%$; $P=0.02$ ), median PFS (not reached vs. 12.66 months; $P=0.013$ ), and median OS (not reached vs. 22.0 months; $P=0.004)$ were all significantly improved with nimotuzumab plus chemoradiotherapy versus chemoradiotherapy alone. With nimotuzumab plus radiotherapy, the RR was $76 \%$ versus $40 \%$ for radiotherapy alone $(P=0.023)$, while median PFS was 10.1 versus 6.9 months $(P=0.24)$, and median OS was 14.37 versus 12.79 months $(P=0.71)$, respectively. The nimotuzumabrelated AEs in group 1 were asthenia, dizziness, microscopic hematuria, vomiting, and loose stools; fever, chills, pruritus, rash, headache, hypertension, and fluctuation in blood pressure were reported as nimotuzumab-related AEs in group 2. There were four cases of skin reactions in patients receiving nimotuzumab [45]. At 48 months, the addition of nimotuzumab to chemoradiotherapy significantly increased median OS compared with chemoradiotherapy alone ( $47 \%$ vs. $21 \%$; HR, $0.35 ; P=0.01$ ), but not when combined with radiotherapy versus radiotherapy alone (34\% vs. $13 \% ; P=$ not specified) [46].

In a double-blind trial, patients with unresectable locoregional SCCHN $(N=106)$ were assigned randomly to receive first-line therapy with nimotuzumab plus radiotherapy versus placebo plus radiotherapy [47]. Complete RRs were $59.5 \%$ for patients receiving nimotuzumab and radiotherapy versus $34.2 \%$ of patients receiving radiotherapy alone $(P=0.038)$, and median OS was 12.5 months and 9.5 months $(P=0.0491)$, respectively. In a subgroup analysis of patients with EGFR-positive tumors, significant survival benefit was seen with nimotuzumab plus radiotherapy versus radiotherapy alone
(16.5 vs. 7.2 months; $P=0.0038$ ). The three most common AEs considered to be related to nimotuzumab treatment were asthenia, fever, and headache. No cases of skin rash were observed. A small pilot study involving 17 patients with locally advanced SCCHN was conducted to evaluate the combination of nimotuzumab and concurrent chemotherapy [48]. The RR was $76 \%$ and no grade $3-4$ AEs were reported. An ongoing phase II study is being conducted to investigate the combination of nimotuzumab plus cisplatin and radiotherapy for locally advanced SCCHN (NCT00702481), and a phase III study is assessing postoperative concurrent chemoradiotherapy with or without nimotuzumab for locally advanced SCCHN (NCT00957086).

Zalutumumab (Genmab; Copenhagen, Denmark) is a fully human, high-affinity anti-EGFR mAb [49], which has received fast track designation from the Food and Drug Administration for advanced, metastatic, and/or unresectable SCCHN that has progressed following standard platinum-based chemotherapy. In a phase I/II study in 28 patients with metastatic/recurrent SCCHN, zalutumumab was associated with a RR of $7.1 \%$ [50]. The most frequently reported AEs were infusion-related reactions, rash/acne, and dyspnea. In a phase III pivotal trial, zalutumumab plus best supportive care (BSC) was compared with BSC plus optional methotrexate (control group) in 286 patients with metastatic/recurrent SCCHN after failure of platinum-based chemotherapy [51]. The dose of zalutumumab was titrated according to the development of skin rash in individual patients. Median OS was not significantly different between groups (6.7 vs. 5.2 months in the zalutumumab versus control group, respectively; HR, $0.77 ; 95 \% \mathrm{CI}, 0.57-1.05 ; P=0.0648)$, but PFS was significantly prolonged (9.9 vs. 8.4 weeks; HR, 0.63; 95\% CI, $0.47-0.84 ; P=0.0012)$. The three most common AEs ( $\geq 10 \%$ of patients) were rash $(92 \%$ for zalutumumab and BSC versus $0 \%$ for control), anemia (25\% vs. $19 \%$ ), and pyrexia ( $22 \%$ vs. $13 \%$ ). Grade 3-4 AEs that were more common in the zalutumumab group than in the control group included rash, hypomagnesemia, pneumonia, and headache. Results are awaited from a phase I/II trial of zalutumumab plus cisplatin-based chemoradiotherapy as first-line therapy for locally advanced SCCHN (NCT00401401). A phase III study to determine whether the addition of zalutumumab to primary curative radiotherapy increases locoregional control in SCCHN is currently recruiting patients (DAHANCA 19, NCT00496652).

\section{Tyrosine kinase inhibitors (TKIs) targeting EGFR}

Gefitinib (Iressa ${ }^{\circledR}$, AstraZeneca; Wilmington, DE), an oral, small-molecule, reversible EGFR TKI, was the first TKI to reach phase III investigation in SCCHN, but is no longer 
being pursued for this indication due to recent negative study results [4]. In a phase II trial assessing second-line gefitinib $500 \mathrm{mg} /$ day in patients with metastatic/recurrent SCCHN $(N=52)$, the RR was $10.6 \%$, median OS was 8.1 months, and 1-year OS rate was $29.2 \%$ [52]. The most common AEs were diarrhea, skin toxicity, and anorexia. A subsequent phase II trial was conducted to evaluate gefitinib $250 \mathrm{mg} /$ day in patients with recurrent and/or metastatic SCCHN $(N=71)$, with the aim of reducing the incidence of toxicities [53]. One patient achieved a PR. Median OS was 5.5 months and PFS was 1.8 months, while the 1-year OS rate was estimated at $19 \%$. Skin toxicity was reported for $64 \%$ of patients.

When gefitinib $250 \mathrm{mg} /$ day was combined with radiotherapy in a phase I/II study involving 16 patients with locally advanced inoperable SCCHN, the RR was $37.5 \%$ [54]. Neoadjuvant gefitinib in combination with docetaxel, carboplatin, and 5-FU, followed by concurrent docetaxel, radiation therapy, and gefitinib was evaluated in patients with locally advanced SCCHN $(N=62)$ [55]. Following completion of neoadjuvant therapy, the RR was $46 \%$, and following completion of adjuvant therapy, the RR was $80 \%$. The estimated 3-year survival rate was $54 \%$. The most common grade 3-4 AEs reported during the neoadjuvant treatment phase were neutropenia, oral mucositis, and diarrhea, while during the adjuvant phase, these were oral mucositis/esophagitis/dysphagia, anorexia, and fatigue.

Two phase III studies have assessed gefitinib in patients with metastatic/recurrent SCCHN. In patients who had received numerous prior treatments, gefitinib $250 \mathrm{mg}$ /day plus docetaxel was compared with docetaxel alone $(N=270)$ [56]. The study was terminated early, with a reported median OS of 6.8 months for gefitinib plus docetaxel versus 6.0 months for docetaxel alone $(P=0.74)$; median PFS was 3.3 versus 2.2 months $(P=0.18)$. In a separate trial, gefitinib $250 \mathrm{mg} /$ day, gefitinib $500 \mathrm{mg} /$ day, and methotrexate were compared in 486 patients [57]. Neither dose of gefitinib significantly increased median OS compared with methotrexate (gefitinib $250 \mathrm{mg} /$ day, 5.6 months vs. methotrexate, 6.0 months; HR, $1.22 ; 95 \%$ CI, $0.95-1.57 ; P=0.12$; and gefitinib $500 \mathrm{mg} /$ day, 6.7 months vs. methotrexate, 6.0 months; HR, 1.12; 95\% CI, 0.87-1.43; $P=0.39$ ). The RR was $2.7 \%$ for gefitinib $250 \mathrm{mg} / \mathrm{day}$, $7.6 \%$ for gefitinib $500 \mathrm{mg} /$ day, and $3.9 \%$ for methotrexate, with no significant differences between either dose of gefitinib and methotrexate. The three most common AEs ( $\geq 10 \%$ of patients) with gefitinib $250 \mathrm{mg} /$ day, gefitinib $500 \mathrm{mg} /$ day, and methotrexate were rash $(29.1,39.2$, and $4.4 \%)$, diarrhea $(25.9,39.2$, and $11.9 \%)$, and stomatitis $(9.5,13.9$, and $34.6 \%)$.

Erlotinib (Tarceva ${ }^{\circledR}$, Genentech; South San Francisco, CA) is another oral, small-molecule, reversible EGFR TKI that has demonstrated efficacy in patients with SCCHN. In a phase I/II study involving 37 patients with locally advanced SCCHN, erlotinib administered in combination with cisplatin and radiotherapy was associated with a RR of 74\% (all complete responses [CRs]) and 3-year PFS and OS rates of 61 and $72 \%$, respectively [58]. The most common nonhematologic AEs were nausea/vomiting, dysphagia, and stomatitis. In another phase II study, patients with locally advanced SCCHN $(N=128)$ were assigned randomly to receive cisplatin plus radiotherapy or cisplatin plus radiotherapy and erlotinib [59]. An interim analysis of the first 100 patients demonstrated a RR for both treatment arms of $71 \%$ (all CR), and the most common serious AEs were nausea, vomiting, and dehydration.

Erlotinib monotherapy was evaluated in a phase II trial involving 155 patients with metastatic/recurrent SCCHN [60]. The RR was $4.3 \%$, median OS was 6.0 months, and median PFS was 9.6 weeks. The most common drugrelated AEs were rash, diarrhea, and dry skin. A separate phase I/II study was conducted to evaluate erlotinib plus cisplatin in patients with metastatic/recurrent SCCHN $(N=51)$ [61]. Of 43 evaluable patients, nine demonstrated a tumor response, the median PFS was 3.3 months, and the median OS was 7.9 months. The three most frequently reported AEs were rash, hypomagnesemia, and anemia.

In ongoing phase II studies, erlotinib in combination with cisplatin-based chemotherapy is being evaluated in chemonaive metastatic/recurrent SCCHN (NCT01064479, NCT00076310); as first-line therapy in combination with cetuximab, carboplatin, and paclitaxel for metastatic/recurrent SCCHN (NCT01316757); as first-line therapy in combination with chemoradiotherapy for locally advanced SCCHN (NCT00720304; NCT00442455); and as first-line therapy in combination with radiotherapy for locally advanced SCCHN (NCT01192815). Phase III studies of erlotinib in combination with first-line platinum-based chemotherapy for metastatic/recurrent SCCHN (NCT00448240) and as maintenance therapy in fully resected SCCHN (NCT00412217) were terminated due to low enrollment.

Lapatinib $\left(\right.$ Tykerb $^{\circledR}$, GlaxoSmithKline; Research Triangle Park, NC, USA) is an oral, small-molecule, reversible inhibitor of both EGFR and ErbB2/HER2 [62]. In a phase I dose-escalation study involving 31 patients with locally advanced SCCHN, lapatinib was administered in combination with cisplatin and radiotherapy [63]. The RR was $81 \%$ for all doses combined (500, 1,000, and $1,500 \mathrm{mg} /$ day) and $65 \%$ at the recommended phase II dose of $1,500 \mathrm{mg} /$ day. The most common AEs $(\geq 10 \%$ of patients) at $1,500 \mathrm{mg} /$ day were radiation mucositis, radiation dermatitis, nausea, and vomiting. In a subsequent phase II trial, lapatinib was again combined with cisplatin and radiotherapy, followed by maintenance lapatinib or placebo, after completion of chemoradiotherapy in patients with locally advanced SCCHN $(N=67)$ [64]. 
The complete RR was $53 \%$ with lapatinib versus $36 \%$ with placebo, and HRs for PFS and OS were 0.71 (95\% CI, $0.34-1.52)$ and 0.70 (95\% CI, 0.31-1.63), respectively. No grade 3-4 AEs were observed during maintenance therapy, other than grade 3 localized edema (lapatinib, 3\%) and weight loss (placebo, 3\%).

In a separate phase II study of lapatinib monotherapy in patients with metastatic/recurrent SCCHN $(N=42)$, no objective responses were observed [65]. In ongoing phase II studies, lapatinib plus capecitabine is being evaluated in metastatic/recurrent SCCHN (NCT01044433), and lapatinib plus radiotherapy is being evaluated in patients with locally advanced SCCHN who cannot tolerate chemotherapy (NCT00490061). In addition, a phase III study is being conducted to investigate lapatinib versus placebo administered postoperatively in combination with chemoradiotherapy followed by maintenance lapatinib/placebo in high-risk patients (NCT00424255).

A key limitation of currently available EGFR-targeted therapies, including mAbs and TKIs, is de novo or acquired resistance, mediated through mechanisms including the mutant type III variant of EGFR [66, 67], mutations in the tyrosine kinase domain of EGFR [67], and tumor cell surface expression of other members of the ErbB receptor family [68]. In an effort to address this issue, TKIs that block more than one member of the ErbB family and/or bind irreversibly to their targets are being investigated for the treatment of SCCHN.

Afatinib (BIBW 2992, Boehringer Ingelheim; Ingelheim, Germany) is an oral, small-molecule, irreversible ErbB family inhibitor that targets EGFR, ErbB2, and ErbB4 [69, 70]. Preliminary results from stage 1 of a 2-stage phase II study of afatinib versus cetuximab in 124 patients with platinum-refractory metastatic/recurrent SCCHN showed PRs in 22 and 13\% of patients, respectively [71]. Median PFS was 16 versus 10 weeks for afatinib versus cetuximab, respectively. Primary afatinibrelated AEs were diarrhea and skin-related AEs, while skin-related AEs were the primary cetuximab-related AEs. A phase III trial of afatinib versus methotrexate in patients with platinum-refractory metastatic/recurrent SCCHN (NCT01345682) is planned, and a phase III trial of afatinib versus placebo as adjuvant therapy after chemoradiotherapy in patients with unresected locoregional SCCHN (NCT01345669) is recruiting participants.

PF-00299804 (PF-299, Pfizer; New York, NY, USA) is an oral, small-molecule, irreversible, pan-HER inhibitor that targets EGFR, ErbB2, and ErbB4 [72]. Results from the first stage of a 2-stage phase II study investigating PF00299804 as first-line treatment in metastatic/recurrent SCCHN showed PRs in 6 of $56(11 \%)$ evaluable patients, and median PFS of 2.8 months. The most common grade 3 AEs ( $\geq 3 \%$ of patients) were diarrhea, fatigue, dermatitis acneiform, and hand-foot skin reaction [73]. The criteria for proceeding to stage 2 of the trial were fulfilled and patient accrual is ongoing.

In addition to treatment strategies targeting more than one ErbB receptor family member and/or binding irreversibly, approaches that combine agents with different mechanisms of action, i.e., targeting other pathways involved in SCCHN, may also have potential to delay or overcome resistance to EGFRtargeted therapy in SCCHN [67]. Preclinical data support the evaluation of the mammalian target of rapamycin (mTOR) pathway, which is involved in EGFR downstream signaling, as a potential therapeutic strategy for SCCHN [74, 75]. A number of clinical trials are currently investigating such treatment combinations. Two phase II studies are evaluating erlotinib plus temsirolimus (NCT01009203) or everolimus (NCT00942734) in platinum-refractory SCCHN. Cetuximab plus temsirolimus is being evaluated in a phase II study in patients with metastatic/recurrent SCCHN not responding to prior cetuximab-based therapy (NCT01256385). Other phase I/II studies are evaluating cetuximab plus platinum and everolimus (NCT01283334), or temsirolimus (NCT01015664), in metastatic/recurrent SCCHN. Cetuximab with cisplatin/paclitaxel plus everolimus or placebo is also being investigated in a phase II study in locally advanced SCCHN (NCT01133678).

High levels of vascular endothelial growth factor (VEGF) expression and some subtypes of VEGF receptors (VEGFRs) $[76,77]$ that are associated with tumor angiogenesis, lymphangiogenesis, and an increased risk of mortality have been observed in SCCHN tumors [76-78]. Results from a phase I/II study of erlotinib plus the anti-VEGFR mAb bevacizumab in patients with metastatic/recurrent SCCHN $(N=48)$ demonstrated an RR of $15 \%$, a median PFS of 4.1 months, and a median OS of 7.1 months [79]. The most common AEs ( $\geq 10 \%$ of patients) were rash, diarrhea, and fatigue. Ongoing phase II studies are evaluating erlotinib plus bevacizumab (NCT00392665) or cetuximab plus bevacizumab (NCT00409565, NCT00407810) in patients with metastatic/recurrent SCCHN, and cetuximab with bevacizumab plus chemoradiotherapy (NCT00703976, NCT00968435) for locally advanced SCCHN. Sorafenib, an inhibitor of multiple protein kinases, including those associated with VEGFRs, is also being evaluated in a phase II study for SCCHN in combination with cetuximab (NCT00815295).

\section{Conclusions}

While several advances have been made in recent decades related to the treatment of locally advanced and metastatic SCCHN, the modest improvements in survival indicate that new treatment strategies are needed. The accumulating data with cetuximab validate the use of anti-EGFR-targeted therapy in this patient group. Several EGFR-targeted 
treatment strategies beyond cetuximab are also being evaluated in phase II and phase III clinical studies for SCCHN, and results from large ongoing clinical trials are awaited. As results of clinical studies mature, greater insight into the potential placement of these agents into the treatment paradigm in SCCHN may be revealed. Validated biomarkers with the potential to predict treatment activity and/or resistance may also contribute to improved patient outcomes in SCCHN.

Acknowledgments This work was supported by Boehringer Ingelheim Pharmaceuticals, Inc. (BIPI). Writing and editorial assistance was provided by Staci Heise, PhD of MedErgy, which was contracted by BIPI for these services. The author meets criteria for authorship as recommended by the International Committee of Medical Journal Editors (ICMJE), is fully responsible for all content and editorial decisions, and was involved at all stages of manuscript development. The author received no compensation related to the development of the manuscript.

Conflict of interest Dr. Agulnik reports previously receiving remuneration for participation in speakers' bureaus on behalf of both Bristol-Myers Squibb and Novartis.

Open Access This article is distributed under the terms of the Creative Commons Attribution Noncommercial License which permits any noncommercial use, distribution, and reproduction in any medium, provided the original author(s) and source are credited.

\section{References}

1. Jemal A, Siegel R, Xu J, Ward E. Cancer statistics, 2010. CA Cancer J Clin. 2010;60:277-300.

2. Sanderson RJ, Ironside JA. Squamous cell carcinomas of the head and neck. BMJ. 2002;325:822-7.

3. National Comprehensive Cancer Network. NCCN clinical practice guidelines in oncology ${ }^{\mathrm{TM}}$. Head and neck cancers. Version 2.2011. http://www.nccn.org/professionals/physician_gls/PDF/headand-neck.pdf. 2011.

4. Fung C, Grandis JR. Emerging drugs to treat squamous cell carcinomas of the head and neck. Expert Opin Emerg Drugs. 2010;15:355-73.

5. Venook AP. Epidermal growth factor receptor-targeted treatment for advanced colorectal carcinoma. Cancer. 2005;103:2435-46.

6. Grandis JR, Tweardy DJ. Elevated levels of transforming growth factor alpha and epidermal growth factor receptor messenger RNA are early markers of carcinogenesis in head and neck cancer. Cancer Res. 1993;53:3579-84.

7. Rubin Grandis J, et al. Levels of TGF-alpha and EGFR protein in head and neck squamous cell carcinoma and patient survival. J Natl Cancer Inst. 1998;90:824-32.

8. Ang KK, et al. Impact of epidermal growth factor receptor expression on survival and pattern of relapse in patients with advanced head and neck carcinoma. Cancer Res. 2002;62:7350-6.

9. Psyrri A, et al. Quantitative determination of nuclear and cytoplasmic epidermal growth factor receptor expression in oropharyngeal squamous cell cancer by using automated quantitative analysis. Clin Cancer Res. 2005;11:5856-62.

10. Dassonville O, et al. Expression of epidermal growth factor receptor and survival in upper aerodigestive tract cancer. J Clin Oncol. 1993;11:1873-8.
11. Chung $\mathrm{CH}$, et al. Increased epidermal growth factor receptor gene copy number is associated with poor prognosis in head and neck squamous cell carcinomas. J Clin Oncol. 2006;24:4170-6.

12. Temam S, et al. Epidermal growth factor receptor copy number alterations correlate with poor clinical outcome in patients with head and neck squamous cancer. J Clin Oncol. 2007;25: 2164-70.

13. Sheridan MT, O'Dwyer T, Seymour CB, Mothersill CE. Potential indicators of radiosensitivity in squamous cell carcinoma of the head and neck. Radiat Oncol Investig. 1997;5:180-6.

14. Chiang WF, et al. Association of epidermal growth factor receptor (EGFR) gene copy number amplification with neck lymph node metastasis in areca-associated oral carcinomas. Oral Oncol. 2008;44:270-6.

15. ERBITUX ${ }^{\circledR}$ (cetuximab) injection, for intravenous infusion [package insert]. Distributed and marketed by Bristol-Myers Squibb Company, Princeton; Co-marketed by Eli Lilly and Company, Indianapolis; 2011.

16. Bonner JA, et al. Radiotherapy plus cetuximab for squamous-cell carcinoma of the head and neck. N Engl J Med. 2006;354:567-78.

17. Bonner JA, et al. Radiotherapy plus cetuximab for locoregionally advanced head and neck cancer: 5-year survival data from a phase 3 randomised trial, and relation between cetuximabinduced rash and survival. Lancet Oncol. 2010;11:21-8.

18. Ang KK, et al. A randomized phase III trial (RTOG 0522) of concurrent accelerated radiation plus cisplatin with or without cetuximab for stage III-IV head and neck squamous cell carcinomas (HNC). J Clin Oncol. 2011;29. Abstract 5500.

19. Pfister DG, et al. Concurrent cetuximab, cisplatin, and concomitant boost radiotherapy for locoregionally advanced, squamous cell head and neck cancer: a pilot phase II study of a new combined-modality paradigm. J Clin Oncol. 2006;24:1072-8.

20. Langer CJ, Li JW, Patel UA, et al. Preliminary analysis of ECOG 3303: concurrent radiation (RT), cisplatin (DDP) and cetuximab (C) in unresectable, locally advanced (LA) squamous cell carcinoma of the head and neck (SCCHN). J Clin Oncol. 2008;26. Abstract 6006.

21. Burtness B, Goldwasser MA, Flood W, Mattar B, Forastiere AA. Phase III randomized trial of cisplatin plus placebo compared with cisplatin plus cetuximab in metastatic/recurrent head and neck cancer: an Eastern Cooperative Oncology Group study. J Clin Oncol. 2005;23:8646-54.

22. Vermorken JB, et al. Platinum-based chemotherapy plus cetuximab in head and neck cancer. N Engl J Med. 2008;359:1116-27.

23. Rozzi A, Lenci G, Corona M, et al. Weekly regimen of paclitaxel-carboplatin-cetuximab as first-line chemotherapy in patients with platinum-resistant recurrent or metastatic squamous cell carcinoma of head and neck (SCCHN): results of a phase II study. Ann Oncol. 2010;21:viii323. Abstract 1035P.

24. Baselga J, et al. Phase II multicenter study of the antiepidermal growth factor receptor monoclonal antibody cetuximab in combination with platinum-based chemotherapy in patients with platinum-refractory metastatic and/or recurrent squamous cell carcinoma of the head and neck. J Clin Oncol. 2005;23:5568-77.

25. Herbst RS, et al. Phase II multicenter study of the epidermal growth factor receptor antibody cetuximab and cisplatin for recurrent and refractory squamous cell carcinoma of the head and neck. J Clin Oncol. 2005;23:5578-87.

26. Vermorken JB, et al. Open-label, uncontrolled, multicenter phase II study to evaluate the efficacy and toxicity of cetuximab as a single agent in patients with recurrent and/or metastatic squamous cell carcinoma of the head and neck who failed to respond to platinum-based therapy. J Clin Oncol. 2007;25:2171-7.

27. Tejani MA, Cohen RB, Mehra R. The contribution of cetuximab in the treatment of recurrent and/or metastatic head and neck cancer. Biologics. 2010;4:173-85. 
28. Moon C, Chae YK, Lee J. Targeting epidermal growth factor receptor in head and neck cancer: lessons learned from cetuximab. Exp Biol Med (Maywood). 2010;235:907-20.

29. Argiris A, Karamouzis MV, Raben D, Ferris RL. Head and neck cancer. Lancet. 2008;371:1695-709.

30. Pignon JP, le Maitre A, Maillard E, Bourhis J. Meta-analysis of chemotherapy in head and neck cancer (MACH-NC): an update on 93 randomised trials and 17,346 patients. Radiother Oncol. 2009;92:4-14.

31. Dirix P, Nuyts S. Evidence-based organ-sparing radiotherapy in head and neck cancer. Lancet Oncol. 2010;11:85-91.

32. Forastiere AA, et al. Randomized comparison of cisplatin plus fluorouracil and carboplatin plus fluorouracil versus methotrexate in advanced squamous-cell carcinoma of the head and neck: a Southwest Oncology Group study. J Clin Oncol. 1992;10: 1245-51.

33. Jacobs C, et al. A phase III randomized study comparing cisplatin and fluorouracil as single agents and in combination for advanced squamous cell carcinoma of the head and neck. J Clin Oncol. 1992;10:257-63.

34. Gibson MK, et al. Randomized phase III evaluation of cisplatin plus fluorouracil versus cisplatin plus paclitaxel in advanced head and neck cancer (E1395): an intergroup trial of the Eastern Cooperative Oncology Group. J Clin Oncol. 2005;23:3562-7.

35. Janinis J, et al. Combination chemotherapy with docetaxel, cisplatin, and 5-fluorouracil in previously treated patients with advanced/recurrent head and neck cancer: a phase II feasibility study. Am J Clin Oncol. 2000;23:128-31.

36. Shin DM, et al. Phase II trial of paclitaxel, ifosfamide, and cisplatin in patients with recurrent head and neck squamous cell carcinoma. J Clin Oncol. 1998;16:1325-30.

37. Shin DM, et al. Phase II study of paclitaxel, ifosfamide, and carboplatin in patients with recurrent or metastatic head and neck squamous cell carcinoma. Cancer. 2001;91:1316-23.

38. Jemal A, et al. Annual report to the nation on the status of cancer, 1975-2001, with a special feature regarding survival. Cancer. 2004;101:3-27.

39. Wirth LJ, et al. Phase I dose-finding study of paclitaxel with panitumumab, carboplatin and intensity-modulated radiotherapy in patients with locally advanced squamous cell cancer of the head and neck. Ann Oncol. 2010;21:342-7.

40. Rischin D, Spigel DR, Adkins D, et al. Panitumumab (pmab) regimen in second-line monotherapy (PRISM) in patients (pts) with recurrent $(\mathrm{R})$ or metastatic (M) squamous cell carcinoma of the head and neck (SCCHN): interim safety analysis. Ann Oncol. 2010;21:viii323. Abstract 1036P.

41. Vermorken JB, Stohlmacher J, Davidenko I, et al. Primary efficacy and safety results of SPECTRUM, a phase 3 trial in patients (pts) with recurrent and/or metastatic (R/M) squamous cell carcinoma of the head and neck (SCCHN) receiving chemotherapy with or without panitumumab (pmab). Ann Oncol. 2010;21: viii12. Abstract LBA26.

42. Mateo $\mathrm{C}$, et al. Humanization of a mouse monoclonal antibody that blocks the epidermal growth factor receptor: recovery of antagonistic activity. Immunotechnology. 1997;3:71-81.

43. Rojo F, et al. Pharmacodynamic trial of nimotuzumab in unresectable squamous cell carcinoma of the head and neck: a SENDO Foundation study. Clin Cancer Res. 2010;16:2474-82.

44. Crombet $\mathrm{T}$, et al. Use of the humanized anti-epidermal growth factor receptor monoclonal antibody h-R3 in combination with radiotherapy in the treatment of locally advanced head and neck cancer patients. J Clin Oncol. 2004;22:1646-54.

45. Ramakrishnan MS, et al. Nimotuzumab, a promising therapeutic monoclonal for treatment of tumors of epithelial origin. MAbs. 2009; $1: 41-8$.
46. Babu KG, Viswanath L, Reddy BK, et al. An open-label, randomized study of h-R3mAb (nimotuzumab) in patients with advanced (stage III or IVa) squamous cell carcinoma of head and neck (SCCHN): four-year survival results from a phase IIb study. J Clin Oncol. 2010;28. Abstract 5530.

47. Rodriguez MO, et al. Nimotuzumab plus radiotherapy for unresectable squamous-cell carcinoma of the head and neck. Cancer Biol Ther. 2010;9:343-9.

48. Gupta M, Madholia V, Gupta N, Bhowmik KT. Results from a pilot study of nimotuzumab with concurrent chemoradiation in patients with locally advanced squamous cell carcinoma of head and neck. J Clin Oncol. 2010;28. Abstract 5565.

49. Bleeker WK, et al. Dual mode of action of a human anti-epidermal growth factor receptor monoclonal antibody for cancer therapy. J Immunol. 2004;173:4699-707.

50. Bastholt L, et al. Phase I/II clinical and pharmacokinetic study evaluating a fully human monoclonal antibody against EGFr (HuMax-EGFr) in patients with advanced squamous cell carcinoma of the head and neck. Radiother Oncol. 2007;85:24-8.

51. Machiels JP, et al. Zalutumumab plus best supportive care versus best supportive care alone in patients with recurrent or metastatic squamous-cell carcinoma of the head and neck after failure of platinum-based chemotherapy: an open-label, randomised phase 3 trial. Lancet Oncol. 2011;12:333-43.

52. Cohen EE, et al. Phase II trial of ZD1839 in recurrent or metastatic squamous cell carcinoma of the head and neck. J Clin Oncol. 2003;21:1980-7.

53. Cohen EE, et al. Phase II trial of gefitinib $250 \mathrm{mg}$ daily in patients with recurrent and/or metastatic squamous cell carcinoma of the head and neck. Clin Cancer Res. 2005;11:8418-24.

54. Caponigro F, et al. A phase I/II trial of gefitinib and radiotherapy in patients with locally advanced inoperable squamous cell carcinoma of the head and neck. Anticancer Drugs. 2008;19:739-44.

55. Hainsworth JD, et al. Neoadjuvant chemotherapy/gefitinib followed by concurrent chemotherapy/radiation therapy/gefitinib for patients with locally advanced squamous carcinoma of the head and neck. Cancer. 2009;115:2138-46.

56. Argiris A, Ghebremichael M, Gilbert J, et al. A phase III randomized, placebo-controlled trial of docetaxel (D) with or without gefitinib $(\mathrm{G})$ in recurrent or metastatic $(\mathrm{R} / \mathrm{M})$ squamous cell carcinoma of the head and neck (SCCHN): a trial of the Eastern Cooperative Oncology Group. J Clin Oncol. 2009;27. Abstract 6011.

57. Stewart JS, et al. Phase III study of gefitinib compared with intravenous methotrexate for recurrent squamous cell carcinoma of the head and neck [corrected]. J Clin Oncol. 2009;27:1864-71.

58. Herchenhorn D, et al. Phase I/II study of erlotinib combined with cisplatin and radiotherapy in patients with locally advanced squamous cell carcinoma of the head and neck. Int J Radiat Oncol Biol Phys. 2010;78:696-702.

59. Hayes DN, Raez LE, Sharma AK, et al. Multicenter randomized phase II trial of combined radiotherapy and cisplatin with or without erlotinib in patients with locally advanced squamous cell carcinoma of the head and neck (SCCAHN): preliminary toxicity results. J Clin Oncol. 2010;28. Abstract 5580.

60. Soulieres D, et al. Multicenter phase II study of erlotinib, an oral epidermal growth factor receptor tyrosine kinase inhibitor, in patients with recurrent or metastatic squamous cell cancer of the head and neck. J Clin Oncol. 2004;22:77-85.

61. Siu LL, et al. Phase I/II trial of erlotinib and cisplatin in patients with recurrent or metastatic squamous cell carcinoma of the head and neck: a Princess Margaret Hospital phase II consortium and National Cancer Institute of Canada Clinical Trials Group Study. J Clin Oncol. 2007;25:2178-83.

62. Kondo N, et al. Antitumor effects of lapatinib (GW572016), a dual inhibitor of EGFR and HER-2, in combination with cisplatin 
or paclitaxel on head and neck squamous cell carcinoma. Oncol Rep. 2010;23:957-63.

63. Harrington KJ, et al. Phase I study of lapatinib in combination with chemoradiation in patients with locally advanced squamous cell carcinoma of the head and neck. J Clin Oncol. 2009;27: $1100-7$.

64. Harrington KJ, Berrier A, Robinson M, et al. Phase II study of oral lapatinib, a dual-tyrosine kinase inhibitor, combined with chemoradiotherapy (CRT) in patients (pts) with locally advanced, unresected squamous cell carcinoma of the head and neck (SCCHN). J Clin Oncol. 2010;28. Abstract 5505.

65. Abidoye OO, Cohen EE, Wong SJ, et al. A phase II study of lapatinib (GW572016) in recurrent/metastatic (R/M) squamous cell carcinoma of the head and neck (SCCHN). J Clin Oncol. 2006;24. Abstract 5568.

66. Sok JC, et al. Mutant epidermal growth factor receptor (EGFRvIII) contributes to head and neck cancer growth and resistance to EGFR targeting. Clin Cancer Res. 2006;12:5064-73.

67. Chen LF, Cohen EE, Grandis JR. New strategies in head and neck cancer: understanding resistance to epidermal growth factor receptor inhibitors. Clin Cancer Res. 2010;16:2489-95.

68. Erjala K, et al. Signaling via ErbB2 and ErbB3 associates with resistance and epidermal growth factor receptor (EGFR) amplification with sensitivity to EGFR inhibitor gefitinib in head and neck squamous cell carcinoma cells. Clin Cancer Res. 2006; 12:4103-11.

69. Li D, et al. BIBW2992, an irreversible EGFR/HER2 inhibitor highly effective in preclinical lung cancer models. Oncogene. 2008;27:4702-11.

70. Yamamoto N, et al. A phase II trial of afatinib (BIBW 2992) in patients (pts) with advanced non-small cell lung cancer previously treated with erlotinib or gefitinib. Poster presented at: the Annual Meeting of the American Society of Clinical Oncology; 4-8 June, 2011; Chicago, IL. Abstract 7524.

71. Seiwert TY, Fayette J, Del Campo JM, et al. A randomized, openlabel phase II study of afatinib (BIBW 2992) versus cetuximab in patients with metastatic or recurrent squamous cell carcinoma of the head and neck (HNSCC) after failure of platinum-containing therapy with a cross-over for progressing patients. Presented at: the 35th European Society for Medical Oncology (ESMO); 8-12 October, 2010; Milan. Abstract 1010PD.

72. Engelman JA, et al. PF00299804, an irreversible pan-ERBB inhibitor, is effective in lung cancer models with EGFR and ERBB2 mutations that are resistant to gefitinib. Cancer Res. 2007;67:11924-32.

73. Siu LL, et al. Phase II trial of the irreversible oral pan-human EGF receptor (HER) inhibitor PF-00299804 (PF) as first-line treatment in recurrent and/or metastatic (RM) squamous cell carcinoma of the head and neck (SCCHN). J Clin Oncol. 2011;29. Abstract 5561.

74. Amornphimoltham $\mathrm{P}$, et al. Mammalian target of rapamycin, a molecular target in squamous cell carcinomas of the head and neck. Cancer Res. 2005;65:9953-61.

75. Aissat N, et al. Antiproliferative effects of rapamycin as a single agent and in combination with carboplatin and paclitaxel in head and neck cancer cell lines. Cancer Chemother Pharmacol. 2008;62:305-13.

76. Neuchrist $\mathrm{C}$, et al. Vascular endothelial growth factor $\mathrm{C}$ and vascular endothelial growth factor receptor 3 expression in squamous cell carcinomas of the head and neck. Head Neck. 2003;25:464-74.

77. Kyzas PA, Cunha IW, Ioannidis JP. Prognostic significance of vascular endothelial growth factor immunohistochemical expression in head and neck squamous cell carcinoma: a metaanalysis. Clin Cancer Res. 2005;11:1434-40.

78. Kyzas PA, Stefanou D, Batistatou A, Agnantis NJ. Prognostic significance of VEGF immunohistochemical expression and tumor angiogenesis in head and neck squamous cell carcinoma. J Cancer Res Clin Oncol. 2005;131:624-30.

79. Cohen EE, et al. Erlotinib and bevacizumab in patients with recurrent or metastatic squamous-cell carcinoma of the head and neck: a phase I/II study. Lancet Oncol. 2009;10:247-57. 